\title{
The value of bilateral simultaneous nasal spirometry in the assessment of patients undergoing septoplasty*
}

\author{
Georgios Fyrmpas ${ }^{1}$, Dionysios Kyrmizakis ${ }^{2}$, Victor Vital ${ }^{1}$, Jannis Constantinidis ${ }^{1}$ \\ 1st Academic Department of Otolaryngology Head \& Neck Surgery, Aristotle University of Thessaloniki, \\ AHEPA Hospital, Kiriakidi 1, 54636 Thessaloniki, Greece \\ 2 Department of Otolaryngology, District General Hospital of Veria, Asomaton, 59100 Veria, Greece
}

SUMMARY

Problem: There is no consensus regarding the best approach to select patients for septoplasty. Patient dissatisfaction after septoplasty implies that clinical examination alone is inadequate to detect a clinically relevant nasal septal deviation (NSD). Objective testing provides an in-depth analysis of nasal obstruction and its underlying anatomic causes but it is effort consuming, requires training and it is not widely available.

Aim: We studied the role of bilateral simultaneous nasal spirometry (BSNS) in the preoperative selection of patients for septoplasty.

Patients, subjects and methods: Thirty patients with nasal obstruction and NSD were assessed by subjective measures and BSNS before and after septoplasty. The decongested nasal partitioning ratio (NPR) was used as a measure of the degree of NSD. Thirty healthy controls were recruited for providing a normal range of NPR values.

Results: All patients were subjectively improved after septoplasty but only those with NPR out of the normal limits had a significant reduction of NPR. Patients with unilateral symptoms and NPR beyond normal limits were also able to identify the more obstructed side preoperatively. For this group of patients, physicians were able to identify the convex side of NSD preoperatively. No correlation between subjective measures of nasal obstruction or airflow asymmetry and NPR was observed.

Conclusion: BSNS is a rapid, easily interpretable, noninvasive technique, which identifies patients with large NSDs who, irrespective of concomitant mucosal factors of nasal obstruction, warrant septoplasty. BSNS is not applicable in cases with a septal perforation or an S type septal deviation, it cannot detect an insufficient nasal valve and it does not substitute rhinomanometry or acoustic rhinometry.

Key words: nasal spirometry, nasal partitioning ratio, nasal septal deviation, septoplasty, patient selection

\section{INTRODUCTION}

Nasal obstruction is a common symptom affecting $9.5-15 \%$ of the general population ${ }^{(1)}$. Mucosal and structural factors of the nose mainly contribute to the pathogenesis of nasal obstruction. Deformities at the nasal valve region including anterior nasal septal deviations (NSD) are the commonest structural problems leading to the subjective sensation of decreased airflow. Septal surgery aims to restore nasal patency by decreasing the nasal resistance in this critical area and address the patients' symptoms ${ }^{(2)}$. Although septoplasty is a common procedure, objective evidence of a clear benefit in adults who present with a non traumatic deviated septum is lacking ${ }^{(3)}$. Twenty seven percent of patients undergoing septal surgery do not improve and $22 \%$ of patients develop a dry nasal mucosa with crust formation postoperatively ${ }^{(4)}$. Nasal obstruction may persist despite successful surgery or may improve spontaneously without any surgery at all (5). Selection of patients who are likely to benefit from septoplasty is therefore of paramount importance. Clinical evaluation of a NSD is unreliable. Septal deformities occur in $1-80 \%$ of the population ${ }^{(3)}$ and this wide variation reflects the subjective nature and the lack of a standardised method of clinical assessment. Acoustic rhinometry (AR) and rhinomanometry (RM) are validated objective tests used in the evaluation of the compromised nasal airway ${ }^{(6)}$. However, widespread use of these techniques in clinical practice is not established yet ${ }^{(7)}$. Nasal spirometry is a simple and quick alternative method to assess nasal patency objectively. To 
date, there are only a few reports on the use of peak nasal inspiratory flow (PNIF) in the management of patients with a NSD ${ }^{(8,9)}$. A modification of nasal spirometry, the bilateral simultaneous nasal spirometry (BSNS), was developed as a simple and practical method of measuring the degree of NSD $(10,11)$. According to this technique, the patient breathes in or out slowly through two separate nosepieces of a specially designed spirometer (rhinospirometer-GM Instruments, Scotland) until a given volume of air has been reached. A ratio based upon the measured inspiratory or expiratory volumes of the two sides is produced. The nasal partitioning ratio (NPR) reflects the degree of the nasal airflow asymmetry and corresponds to the degree of nasal septal deviation. Values range between -1 (complete left sided obstruction) to +1 (complete right sided obstruction). BSNS correlates well with rhinomanometry ${ }^{(11)}$ and normal reference values for NPR have already been published ${ }^{(12)}$. This method has been evaluated in the study of the nasal cycle ${ }^{(13)}$ and the selection of patients for septoplasty ${ }^{(10,14)}$.

The purpose of this study is to investigate the validity of BSNS as a quick and easy method of selecting patients for septoplasty and assess the ability of the patients to identify the more obstructed side of the nose based on the BSNS measurements.

\section{PATIENTS, SUBJECTS AND METHODS}

\section{General evaluation of patients}

All adult patients presenting with nasal obstruction and a clinically obstructing deviated septum were invited to participate in the study. Patients were examined and recruited by the first author in a tertiary referral center. Patient characteristics are shown in Table 1. Preoperative evaluation included a thorough history including questions for rhinitis triggers such as hormonal problems or medications, clinical examination, allergy testing and sinus $\mathrm{x}$-rays. The anterior nose was assessed by rhinoscopy and the clinical tests for nasal valve collapse. The middle and posterior nasal cavity were assessed by nasal endoscopy. Allergy skin prick tests (Allergopharma allergens) with the 30 most common aeroallergens in Northern Greece were performed in patients with a positive history of exposure and rhinitis symptoms. If allergy tests were negative and the patient suffered from symptoms of rhinitis such as sneezing or rhinorrhea under certain conditions (e.g. perfumes, food, workplace exposure), he or she was categorized as having non allergic rhinitis.

Patients were excluded from the study if they had an S type septal deviation, a septal perforation, nasal polyps, an upper respiratory tract infection in the preceding 2 months and chronic rhinosinusitis. Patients undergoing septorhinoplasty or revision septoplasty or septoplasty not for nasal obstruction (e.g. access to the paranasal sinuses or for improving the application of a continuous positive pressure device) were also excluded. Ethical committee approval and patient informed consent for participation in the study were obtained.
Table 1. Description of study population.

\begin{tabular}{lc}
\hline Demographic characteristics & $\mathrm{n}(\%)$ \\
Patients & 30 \\
Males/females & $25 / 5$ \\
Mean age & 33 years \\
Smokers & $15(50)$ \\
History of nasal trauma & $12(40)$ \\
Rhinitis & $19(63.3)$ \\
Bilateral nasal valve insufficiency & $3(10)$ \\
Treatment characteristics & \\
Septoplasty only & \\
Septoplasty + contralateral ITRF & $(26.7)$ \\
Septoplasty + bilateral ITRF & $4(13.3)$ \\
Total & $18(60)$ \\
ITRF: inferior turbinate reduction by radiofrequency tissue ablation
\end{tabular}

Intervention and postoperative follow up

All patients underwent a typical Cottle's septoplasty with or without reduction of one or both inferior turbinates using radiofrequencies by experienced surgeons (Table 1). No interventions on the nasal valve were performed at this stage. No medications except normal saline irrigations and antibiotics were allowed postoperatively until the follow up examination. The second visit was arranged two months after surgery so that any mucosal disturbance due to the operation would have subsided.

Assessment of nasal obstruction

1) Subjective outcome measures

a) Nasal obstruction was quantified using the specifically designed NOSE scale before decongestion (values ranged from 0 to 100$)$.

b) The degree of asymmetry of nasal airflow after decongestion was expressed by a double vertical 10-point scale (asymmetry of nasal airflow scale) as proposed by Boyce et al. ${ }^{(14)}$. In each vertical column, point ' 1 ' signified that no air could pass through one nostril and point ' 10 ' signified that air passed freely from the nostril. The difference between the two sides was assessed pre and postoperatively.

\section{2) Objective measure}

The degree of NSD, reflected by the NPR, was compared pre and postoperatively after decongestion. Post decongestion measurements are not influenced by the nasal cycle; thus the structural component of the anterior nose (mainly the septum, the conchal bone to a lesser degree) is the only contributor to airflow asymmetry recorded by the rhinospirometer.

\section{Assessment of the more obstructed nasal side}

1) Subjective measures

a) Patient's history: patients were asked to recall which side felt as causing most symptoms before decongestion and without blocking one of the nostrils. Possible answers were 'right', 'left', 'both sides equally' and 'side changes'. In the analysis were only included patients with unilateral complaints. This outcome was recorded only preoperatively as it referred to the long standing 


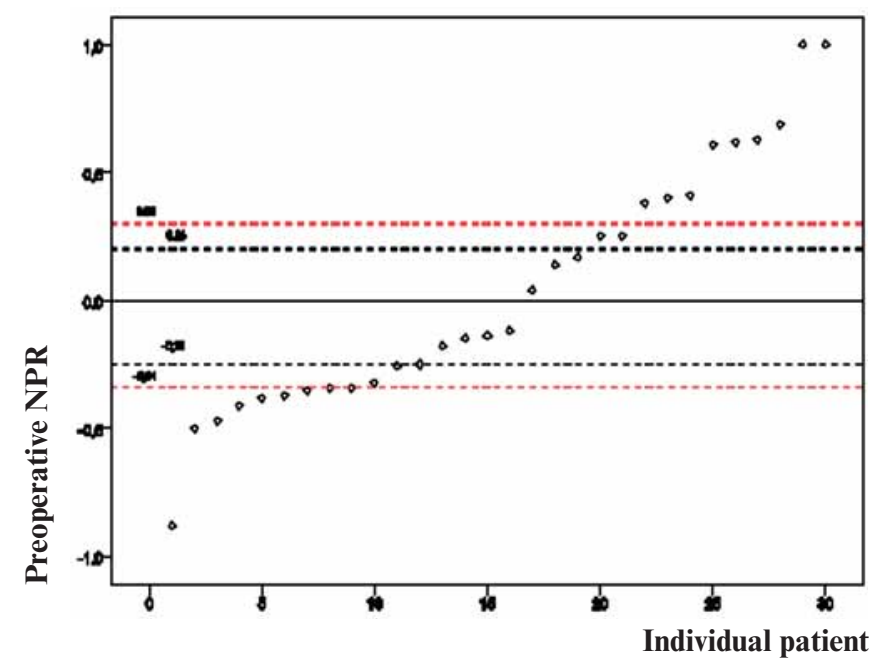

Figure 1. Comparison of preoperative nasal airflow asymmetry and normal reference values. Nasal partitioning ratio (NPR) ranges from - 1 (complete left nasal obstruction) to +1 (complete right nasal obstruction). Black lines: limits derived from our group of asymptomatic patients. Red lines: normal limits according to Roblin et al ${ }^{(12)}$.

patient's perception of nasal obstruction.

b) Asymmetry of nasal airflow scale: The more obstructed side was inferred from the double vertical 10-point scale (the column with the lowest value indicated the side of obstruction). Pre and post operative values were obtained.

c) Clinical examination: The treating physicians were asked to indicate the more obstructed side pre and postoperatively.

\section{2) Objective measures}

The sign of the pre- and postoperative NPR after decongestion indicated the more obstructed side. Negative values signified left- and positive values right-sided obstruction.

\section{Measurement of NPR}

A rhinospirometer was used for measuring simultaneously the airflow in both nasal cavities. The device was calibrated daily and an antiviral/antibacterial filter was used for each individual patient. The patients remained sitting in a room with controlled temperature and humidity. After decongestion, they were asked to breathe slowly in through two nosepieces, which were sealed gently onto the nares. The measurement was completed when 3 liters of air had been inspired from one side and the procedure was repeated three times. The NPR was calculated by the bilateral inspired volumes using appropriate software and the mean NPR of the three measurements was taken into account. For decongestion, cotton pledgets soaked with xylometazoline 1\% were applied between the head of the inferior turbinate and the septum for 15 minutes. Patients were asked to clear their noses before measurements.

\section{Normal reference values}

Thirty healthy individuals were invited to participate in the study for providing normal NPR values. Their history was negative for any nasal symptoms, trauma, interventions, asthma, atopy, recent upper airway infection, medication use or smoking. Subjects were recruited only if the nasal septum was straight on rhinoscopy. A mean value of NPR was calculated after 3 post decongestion measurements. The range of values between the maximum negative and maximum positive NPR values of the healthy group defined the normal reference range. Patients were subdivided into two groups according to the relation of their preoperative NPR values to the normal range (Figure 1). Subgroup 1 included patients with NPR within the normal limits and subgroup 2 all patients outside the normal range. The average NPRs (absolute values) for the two subgroups were compared before and after septoplasty to test if there was a significant change.

\section{Statistical analyses}

Data were analyzed with SPSS 17.0 (SPSS Inc., Chicago, IL, USA). Descriptive statistics of subjective measures of nasal obstruction and NPR were presented as medians with interquartile ranges (IQR) for normally and not normally distributed data to allow direct comparisons. Correlations between the subjective measures of nasal obstruction and NPR measurements were analyzed with the Spearman's correlation coefficient 'rho' due to the skewed distribution of the data. The NPRs of the healthy and the patient group were compared with the Mann-Whitney test. For 'before and after' analysis of continuous variables in each patient subgroup, we utilized the Wilcoxon Signed ranks test. To test the agreement between the different outcome measures of identifying the more obstructed side of the nose (nominal variables), the Cohen's k statistic was used. To examine if there was a significant change of the degree of agreement before and after septoplasty in each group, the McNemar's test was employed (nominal data/before-after analysis of one group). The level of statistical significance was set at 0.05 (two-tailed).

\section{RESULTS}

Postoperative change in symptoms and degree of NSD

\section{1a. All patients}

The mean age of all patients was 33 years (range 17 - 52) and the mean age of the healthy group 31.7 years (range $19-49$ ). No sex difference was noted between patients and healthy individuals. There were 14 patients with a positive NPR (right sided NSD) and 16 patients with a negative NPR (left sided NSD). No postoperative complications were reported.

Healthy group: the median absolute NPR was 0.12 (IQR: 0.8 - 0.17); values ranged from - 0.25 to 0.24 . Patient group: the median absolute preoperative NPR was 0.36 (IQR: 0.23 - 0.52 ) and the median absolute postoperative NPR was 0.15 (IQR: $0.06-0.30$ ). The preoperative NPR of patients was significantly higher than the NPR of healthy individuals $(\mathrm{p}<0.0001)$ but the postoperative NPR of the patients did not differ from the NPR of the healthy individuals significantly $(p=0.206)$. Postoperatively two patients had an increased NPR (surgical failures) and in 9 patients the polarity of NPR reversed, indicating a switch of the more obstructed nasal side.

The degree of nasal obstruction and asymmetrical nasal breathing were significantly reduced post-septoplasty and so 
Table 2. Post-septoplasty change of subjective and objective measures of nasal obstruction.

\begin{tabular}{|c|c|c|c|c|c|c|c|c|c|}
\hline \multirow[b]{2}{*}{$\begin{array}{l}\text { Measure of nasal } \\
\text { obstruction }\end{array}$} & \multicolumn{3}{|c|}{ All patients $(n=30)$} & \multicolumn{3}{|c|}{ Subgroup $1(n=9)$} & \multicolumn{3}{|c|}{ Subgroup $2(n=21)$} \\
\hline & Before & After & $\mathrm{p}$ & Before & After & $\mathrm{p}$ & Before & After & $\mathrm{p}$ \\
\hline 1. NOSE score & $70[60-75]$ & $20[3.7-36.2]$ & $<0.001$ & $70[57.5-85]$ & $30[15-42.5]$ & 0.008 & $70[60-75]$ & $21.9[0-30]$ & $<0.001$ \\
\hline $\begin{array}{l}\text { 2. Asymmetry of } \\
\text { nasal airflow } \\
\text { scale }\end{array}$ & $4[2-2.5]$ & $2[1-5]$ & $<0.001$ & $2[1-3.5]$ & $1.1[0-2]$ & 0.071 & $5[2.5-6]$ & $2[1-3]$ & $<0.001$ \\
\hline 3. NPR & $0.36[0.23-0.52]$ & $0.15[0.06-0.30]$ & $<0.001$ & $0.15[0.13-0.21]$ & $0.05[0.04-0.13]$ & 0.192 & $0.41[0.34-0.62]$ & $0.25[0.13-0.34]$ & $<0.001$ \\
\hline
\end{tabular}

Subgroups 1 and 2 are defined by our normal reference NPR limits. Values in square brackets correspond to interquartile percentiles. NPR: nasal partitioning ratio (absolute values).

was the absolute NPR (Table 2). However, there was no correlation pre or postoperatively of the NOSE scale with the absolute NPR $(p>0.1)$. Neither was the degree of reduction of the NOSE significantly correlated with the improvement of the absolute NPR $(p>0.1)$

\section{1b. Subgroups of patients}

In subgroup 1, 7 out of 9 patients $(77.8 \%)$ suffered from treated or untreated rhinitis and one patient had bilateral nasal valve collapse at the level of the upper laterals and hinge area. Prior nasal trauma reported 2 patients $(22.2 \%)$.

In subgroup 2, 12 out of 21 patients (57.1\%) suffered from rhinitis, two had nasal valve insufficiency and 10 patients reported nasal trauma (47.6\%).

Subjective measures of nasal obstruction decreased significantly for both subgroups after septoplasty except for the degree of nasal airflow asymmetry in subgroup 1 (Table 2). The NPR reduced significantly in subgroup $2(p=0.001)$ but not in subgroup $1(\mathrm{p}=0.192)$.

\section{Identification of the more obstructed side of the nose}

\section{2a. All patients}

Preoperatively, most patients $(92.3 \%)$ were able to identify the convex side of the septum as the side of the nose responsible for their symptoms (Table 3). The obstructed side was also correctly identified after decongestion by the asymmetry of nasal airflow scale $(83.3 \%)$. There was only moderate agreement between the physician's evaluation of the convex side of the septum and the convex side as indicated by the NPR (correct identification $76.6 \%, \mathrm{k}=0.54)$. Postoperatively, the agreement between subjective measures and NPR fell to a fair or moderate level. This change of agreement was not significant between the asymmetry of nasal airflow scale and NPR (Mc Nemar's test, $p=0.180$ ) but it reached statistical significance for the level of agreement between the physician's perception and NPR $(p=0.039)$.

\section{2b. Subgroups of patients}

Preoperatively, there was only moderate agreement between patients of subgroup 1 and NPR and very little agreement between physicians and NPR. On the contrary, all patients in subgroup 2 were able to indicate the convex side of the septum as the side of their unilateral symptoms and the level of agreement was substantial; the asymmetry of nasal airflow scale and the physician's evaluation agreed also with NPR substantially $(\mathrm{k}=0.72$ and $\mathrm{k}=0.71$, respectively). This level of agreement dropped to a fair or moderate level postoperatively as presented in Table 3. The 'before-after' analysis showed that this change did not reach significance neither for the double vertical scale (Mc Nemar's test, $p=0.289$ ) nor for the physicians' clinical assessment (Mc Nemar's test, $\mathrm{p}=0.219$ ).

\section{DISCUSSION}

BSNS can detect a significant reduction of a clinically important nasal septal deviation after surgery. Patients with severe NSDs report symptoms ipsilateral to the deviated side and show a significant improvement of the deviation postoperatively. BSNS is not an objective measure of nasal obstruction because the degree of symptomatic improvement does not correlate to the improvement of the nasal airflow asymmetry.

The perception of a blocked nose is the result of a complex, largely unknown interplay between several factors: compromised nasal patency ${ }^{(15)}$, disturbed airflow characteristics (turbulent vs laminar flow) ${ }^{(15)}$, abnormal trigeminal sensation (16), the presence or absence of a nasal cycle ${ }^{(17)}$ and even personal characteristics of the individual ${ }^{(18)}$. A NSD reduces nasal patency, interferes with airflow characteristics ${ }^{(19)}$ and the nasal cycle ${ }^{(20)}$ and compromises the air-conditioning capacity of the nose ${ }^{(21)}$. Possibly these mechanisms link a deviated septum to the subjective perception of nasal obstruction but they cannot account always for the full extent of patient's complaints. How deviated should be a nasal septum in the context of concomitant pathologies to contribute to the subjective sensation of nasal blockage that will reverse with surgery is not known.

Surgical failures result from 'physiological' deviations ${ }^{(20)}$ being operated and other factors such as rhinitis or nasal valve collapse not being addressed ${ }^{(22)}$. Our study showed that patients with deviations beyond the normal reference range improve objectively and subjectively irrespective of the presence of rhinitis. The same analysis for the normal reference range proposed by Roblin et al. ${ }^{(12)}(+0.30$ to -0.34$)$ yielded the same results. Additionally, nasal trauma was reported by almost half of these patients. In contradistinction, patients with deviations within the normal limits showed subjective improvement but the degree of deviation was not significantly altered. Most of these patients suffered from rhinitis but not a prior nasal trauma. The placebo effect of surgery as shown also by Cuddihy et al. ${ }^{(10)}$ or co-interventions to the inferior turbinates could 
Table 3. Identification of the more obstructed side of the nose before and after septoplasty.

\begin{tabular}{|c|c|c|c|c|c|c|c|c|c|c|c|c|c|c|c|}
\hline \multirow{3}{*}{$\begin{array}{l}\text { Evaluation of } \\
\text { obstructed side }\end{array}$} & \multicolumn{5}{|c|}{ All patients } & \multicolumn{5}{|c|}{ Subgroup 1} & \multicolumn{5}{|c|}{ Subgroup 2} \\
\hline & Patients & \multicolumn{4}{|c|}{ Correct identification $(\%)$} & \multirow{2}{*}{$\begin{array}{c}\text { Patients } \\
\mathrm{n}\end{array}$} & \multicolumn{4}{|c|}{ Correct identification $(\%)$} & \multirow{2}{*}{$\begin{array}{c}\text { Patients } \\
n\end{array}$} & \multicolumn{4}{|c|}{ Correct identification $(\%)$} \\
\hline & $\mathrm{n}$ & Before & $\mathrm{k}$ & After & $\mathrm{k}$ & & Before & $\mathrm{k}$ & After & $\mathrm{k}$ & & Before & $\mathrm{k}$ & After & $\mathrm{k}$ \\
\hline 1. Patient's history & $26^{*}$ & $24(92.3)$ & 0.85 & - & - & 7 & $5(71.4)$ & 0.46 & - & - & $19^{*}$ & $19(100)$ & 1 & - & - \\
\hline $\begin{array}{l}\text { 2. Asymmetry of } \\
\text { nasal airflow scale }\end{array}$ & 30 & $25(83.3)$ & 0.67 & $19(63.3)$ & 0.41 & 9 & $7(77.7)$ & 0.55 & $5(55.6)$ & 0.38 & 21 & $18(85.7)$ & 0.72 & $14(66.7)$ & 0.37 \\
\hline $\begin{array}{l}\text { 3. Clinical } \\
\text { examination }\end{array}$ & 30 & $23(76.6)$ & 0.54 & $15(50)$ & 0.27 & 9 & $5(55.6)$ & 0.05 & $1(11.1)$ & 0.00 & 21 & $18(85.7)$ & 0.71 & $14(66.7)$ & 0.47 \\
\hline $\begin{array}{l}\text { Interpretation of } \\
\text { Kappa }\end{array}$ & $\begin{array}{c}\text { Kappa } \\
\text { Agreement }\end{array}$ & $\begin{array}{l}<0 \\
\text { Less }\end{array}$ & than $\mathrm{cl}$ & tance & & :light & $\begin{array}{r}0.21-0 \\
\text { fail }\end{array}$ & & $\begin{array}{l}0.41-0 \\
\text { moder }\end{array}$ & & $\begin{array}{r}0.61 \\
\text { subst }\end{array}$ & $\begin{array}{l}-0.80 \\
\text { antial }\end{array}$ & & $\begin{array}{r}0.81-0.99 \\
\text { most perfe }\end{array}$ & \\
\hline
\end{tabular}

*patients reporting alternating/bilateral nasal obstruction were excluded from analysis. Agreement was tested between subjective measures of evaluating the more obstructed side and the narrower side according to the NPR sign.

account for the subjective improvement of patients with small NSDs. We tested group differences regarding the frequency of rhinitis, inferior turbinate co-interventions and history of nasal trauma. Although patients with small deviations suffered from rhinitis more often $(77.8 \%$ vs $57.1 \%)$, many of them underwent bilateral inferior turbinate reduction $(66.7 \%$ vs $57.1 \%)$ and only a few reported prior nasal trauma $(22.2 \%$ vs $47.6 \%)$ compared to patients with large deviations, the differences did not reach statistical significance possibly due to small sample size.

Another interesting finding in our study was the postoperative switch of the more obstructed nasal side in nine patients (30\%). Four patients detected the change of the nasal patency correctly. This phenomenon has been reported by other investigators ${ }^{(10,23,24)}$. Long standing NSDs are characterized by a compensatory enlargement of the contralateral inferior turbinate, mainly the bone component ${ }^{(25)}$. Although the mucosal element shrinks post-septoplasty, the bone hypertrophy persists for at least a year ${ }^{(26)}$. If no conchal bone is removed during septoplasty, the nasal cavity on the concave side of a deviated septum narrows and this may account for the observed change of the NPR sign.

Patients with unilateral symptoms can identify correctly the side of obstruction pre or post decongestion better than physicians. The ability to correctly discriminate the more obstructed side of the nose is reduced in all patients after septoplasty and in patients with deviations within the normal limits. Clarke et al. ${ }^{(27)}$ found that if the flow difference between the two sides of the nose exceeds $100 \mathrm{~cm}^{3} / \mathrm{s}, 95 \%$ of patients with acute rhinitis can identify the low flow nasal passage on a $100 \mathrm{~mm}$ VAS. This ability increases for low total nasal resistance suggesting that the nose becomes more sensitive at lower flow rates. Sipila et al. ${ }^{(28)}$ showed that $80.6 \%$ of patients with increased unilateral nasal resistance due to a deviated septum could identify correctly the more obstructed side. Only $50 \%$ of patients with normal nasal resistance yielded the same result. Hirschberg et al. ${ }^{(29)}$ reported that $84 \%$ of patients and healthy subjects were correct in their judgment regardless of nasal resistance. A disturbed nasal cycle causing paradoxical nasal obstruction and a nasal valve compromise should be taken into account when correlating a deviated septum with the perceived side of obstruction. Constantian et al. ${ }^{(30)}$ showed that $54 \%$ of patients with lateralized symptoms failed to identify the convex side of the deviation as the side of obstruction. These patients presumably had a contralateral incompetent nasal valve, which collapsed under the greater airflow on the wide nasal side. The discrepancy between studies on the ability to discriminate the more obstructed side may be explained by differences in populations. Constantian's patients comprised a highly selected group with complex valvular problems and most of these patients had already undergone a septo- or rhinoplasty. Presumably, the main cause of their problems was the nasal valve and not the septum.

Physicians are accurate at identifying the convex side of a 'severe' but not a 'moderate' nasal deviation. The sensitivity and specificity of physician's assessment compared to acoustic rhinometry and rhinomanometry measurements are $55 \%{ }^{(31)}$. Boyce et al. ${ }^{(14)}$ used BSNS and yielded different results with high sensitivity $(100 \%)$ but low specificity $(30 \%)$. Our results also suggest that physicians are able to identify the convex side of a NSD when the deviation exceeds the normal range of NPR values. This ability is reduced postoperatively but the reduction does not reach statistical significance possibly due to small sample size.

Preoperative planning based on subjective assessment of a NSD by anterior rhinoscopy is not sufficient. McCaffrey et al. ${ }^{(32)}$ showed that a deviated septum may coexist with normal nasal resistance. Clinical examination by simple non distorting inspection of the nasal vestibule after quiet or forced inspiration may reveal which elements of the nasal valve, apart from a deviated septum, play a role in the nasal airway obstruction (30). The decongestion test may be useful in predicting a positive outcome after reduction of the inferior turbinates ${ }^{(33)}$.

Objective assessment of patients undergoing surgery for nasal obstruction has been criticized as adding little information to the clinical judgment ${ }^{(7)}$. In the preoperative selection of 
patients with NSD, several criteria based on rhinomanometry have been proposed: flow less than $700 \mathrm{~cm}^{3} / \mathrm{sec}^{(34)}$, total nasal resistance greater than $0.1 \mathrm{~Pa} / \mathrm{cm}^{3} / \mathrm{sec}$ or unilateral nasal resistance greater than $0.35 \mathrm{~Pa} / \mathrm{cm}^{3} / \mathrm{sec}$ and the post decongestion intercavital flow ratio (less than 1:2) ${ }^{(31)}$. Acoustic rhinometry may reveal the mucosal element of nasal obstruction and provides us with a cutoff value of post decongestion MCA at the narrower side of a NSD beyond which patients are likely to benefit from surgery $\left(0.40 \mathrm{~cm}^{2}\right)^{(31)}$. Nasal spirometry has also been used in the management of patients undergoing septoplasty ${ }^{(8,9,36)}$ but no specific preoperative criteria based on PNIF have been proposed so far.

BSNS utilizes a similar technique to spirometry to assess the asymmetrical nasal airflow caused by a deviated septum. The partitioning of nasal airflow as a means of selecting patients for septoplasty was initially proposed by Postema et al. ${ }^{(37)}$. BSNS does not substitute rhinomanometry or acoustic rhinometry but it serves as a quick and reliable means of quantifying objectively the degree of NSD. Additionally, BSNS can identify a group of patients with septal deviations large enough to warrant surgical correction despite the coexistence of mucosal factors contributing to the obstruction. Our results agree with those of Hanif et al. ${ }^{(11)}$ and Cuddihy et al. ${ }^{(10)}$ but the limits of normal references values as proposed by Roblin et al. ${ }^{(12)}$ are different to ours. This may be attributed to our highly selected healthy population and its small size. Hygienic issues regarding BSNS have been resolved with the introduction of single use antiviral/bacterial filters. When BSNS is used in clinical practice certain limitations should be born in mind: a) there is no correlation between the degree of NSD and subjective perception of obstruction b) the normal reference limits of NPR on which the decision to operate is based are not clear cut; thus they may not apply for every individual patient c) nasal valve compromise is not assessed d) $\mathrm{S}$ type septal deformities and septal perforations give unreliable results e) inadequate sealing of the nostrils, excessive pressure upon application of the nosepieces and breathing with effort may distort the anterior nasal skeleton and influence the measurements.

\section{CONCLUSIONS}

A patient with nasal obstruction and a deviated nasal septum presents always a preoperative challenge. Sophisticated methods such as rhinomanometry (4 phase rhinomanometry and rhinoresistometry) and acoustic rhinometry offer a thorough evaluation of patient symptoms and support the decision to operate but they are not widely available. BSNS identifies patients with septal deviations beyond the normal NPR limits who will have a significant postoperative reduction of the deviation. These patients attribute their unilateral complaints to the narrower side of the nose before or after decongestion. For this group of patients, there is agreement between the subjective perception of the more obstructed side and the deviated side on anterior rhinoscopy. BSNS is a simple, quick, noninvasive and reproducible technique, which is easy to interpret and requires minimal training. This test offers valuable information along with clinical assessment of the nasal valve, nasal endoscopy and allergy testing in the preoperative assessment of candidates for septoplasty when other objective methods are not available.

\section{ACKNOWLEDGEMENTS}

We would like to thank Anna Bettina Haidich for reviewing the statistics of this study.

\section{AUTHOR CONTRIBUTIONS}

GF conceived the study topic, prepared the study protocol, acquired, analysed the patient data and wrote the paper. DK took part in the clinical care of the patients and contributed to the evaluation of patients and the preparation of this paper. VVtook part in the clinical care of the patients and contributed to the study protocol. JC contributed to all aspects of the study protocol, took part in the clinical care of the patients and supervised the study and the paper preparation.

The authors declare no conflict of interest.

\section{REFERENCES}

1. Akerlund A, Millqvist E, Oberg D, Bende M. Prevalence of upper and lower airway symptoms: the Skovde population-based study. Acta Otolaryngol. 2006; 126: 483-488.

2. Garcia GJ, Rhee JS, Senior BA, Kimbell JS. Septal deviation and nasal resistance: an investigation using virtual surgery and computational fluid dynamics. Am J Rhinol Allergy. 2010; 24: e46-53.

3. Roblin DG, Eccles R. What, if any, is the value of septal surgery? Clin Otolaryngol Allied Sci. 2002; 27: 77-80.

4. Mlynski G. Impaired function of the upper respiratory tract. Restorative procedures for upper airway dysfunction, nasal breathing. Laryngorhinootologie. 2005; 84 Suppl 1: S101-17.

5. Jessen M, Ivarsson A, Malm L. Nasal airway resistance and symptoms after functional septoplasty: comparison of findings at 9 months and 9 years. Clin Otolaryngol Allied Sci. 1989; 14: 231234.

6. Schumacher MJ. Nasal congestion and airway obstruction: the validity of available objective and subjective measures. Curr Allergy Asthma Rep. 2002; 2: 245-251.

7. Andre RF, Vuyk HD, Ahmed A, Graamans K, Nolst Trenite GJ. Correlation between subjective and objective evaluation of the nasal airway. A systematic review of the highest level of evidence. Clin Otolaryngol. 2009; 34: 518-525.

8. Larsen K, Kristensen S. The peak flow nasal patency index. Ear Nose Throat J. 1992; 71: 23-25.

9. Larsen K, Oxhoj H. Spirometric forced volume measurements in the assessment of nasal patency after septoplasty. A prospective clinical study. Rhinology. 1988; 26: 203-208.

10. Cuddihy PJ, Eccles R. The use of nasal spirometry as an objective measure of nasal septal deviation and the effectiveness of septal surgery. Clin Otolaryngol Allied Sci. 2003; 28: 325-330.

11. Hanif J, Jawad SS, Eccles R. A study to assess the usefulness of a portable spirometer to quantify the severity of nasal septal deviation. Rhinology. 2003; 41: 11-15.

12. Roblin DG, Eccles R. Normal range for nasal partitioning of airflow determined by nasal spirometry in 100 healthy subjects. Am J Rhinol. 2003; 17: 179-183.

13. Hanif J, Eccles R, Jawad SS. Use of a portable spirometer for studies on the nasal cycle. Am J Rhinol 2001; 15: 303-306.

14. Boyce JM, Eccles R. Assessment of subjective scales for selection of patients for nasal septal surgery. Clin Otolaryngol .2006; 31 : 297-302.

15. Mlynski G, Beule A. Diagnostic methods of nasal respiratory 
function. HNO 2008; 56: 81-99.

16. Naclerio RM, Bachert C, Baraniuk JN. Pathophysiology of nasal congestion. Int J Gen Med. 2010; 3: 47-57.

17. Arbour P, Kern EB. Paradoxical nasal obstruction. Can J Otolaryngol 1975; 4: 333-338.

18. Wilson IB, Cleary PD. Linking clinical variables with healthrelated quality of life. A conceptual model of patient outcomes. JAMA. 1995; 273: 59-65.

19. Leong SC, Chen XB, Lee HP, Wang DY. A review of the implications of computational fluid dynamic studies on nasal airflow and physiology. Rhinology. 2010; 48: 139-145.

20. Mlynski G. Surgery of the nasal septum. Facial Plast Surg. 2006; 22: 223-229.

21. Rozsasi A, Leiacker R, Kuhnemann S, et al. The impact of septorhinoplasty and anterior turbinoplasty on nasal conditioning. Am J Rhinol. 2007; 21: 302-306.

22. Becker SS, Dobratz EJ, Stowell N, Barker D, Park SS. Revision septoplasty: review of sources of persistent nasal obstruction. Am J Rhinol. 2008; 22: 440-444.

23. Pirila T, Tikanto J. Unilateral and bilateral effects of nasal septum surgery demonstrated with acoustic rhinometry, rhinomanometry, and subjective assessment. Am J Rhinol. 2001; 15: 127-133.

24. Grymer LF, Hilberg O, Elbrond O, Pedersen OF. Acoustic rhinometry: evaluation of the nasal cavity with septal deviations, before and after septoplasty. Laryngoscope. 1989; 99: 1180-1187.

25. Berger G, Hammel I, Berger R, Avraham S, Ophir D. Histopathology of the inferior turbinate with compensatory hypertrophy in patients with deviated nasal septum. Laryngoscope. 2000; 110: 2100-2105.

26. Kim DH, Park HY, Kim HS, et al. Effect of septoplasty on inferior turbinate hypertrophy. Arch Otolaryngol Head Neck Surg. 2008; 134: 419-423.

27. Clarke JD, Hopkins ML, Eccles R. How good are patients at determining which side of the nose is more obstructed? A study on the limits of discrimination of the subjective assessment of unilateral nasal obstruction. Am J Rhinol. 2006; 20: 20-24.

28. Sipila J, Suonpaa J, Laippala P. Sensation of nasal obstruction compared to rhinomanometric results in patients referred for septoplasty. Rhinology 1994; 32: 141-144.

29. Hirschberg A, Rezek O. Correlation between objective and subjective assessments of nasal patency. ORL J Otorhinolaryngol Relat
Spec. 1998; 60: 206-211.

30. Constantian MB, Clardy RB. The relative importance of septal and nasal valvular surgery in correcting airway obstruction in primary and secondary rhinoplasty. Plast Reconstr Surg. 1996; 98: 38-54; discussion 55-8.

31. Pirila T, Tikanto J. Acoustic rhinometry and rhinomanometry in the preoperative screening of septal surgery patients. Am J Rhinol Allergy. 2009; 23: 605-609.

32. McCaffrey TV, Kern EB. Clinical evaluation of nasal obstruction. A study of 1000 patients. Arch Otolaryngol. 1979; 105: 542-545.

33. Jones AS, Wight RG, Kabil Y, Beckingham E. Predicting the outcome of submucosal diathermy to the inferior turbinates. Clin Otolaryngol Allied Sci. 1989; 14: 41-44.

34. Bachmann W. Differential diagnosis in patients with nasal obstruction: rhinomanometric indications for surgery. 1990; 7: 274

35. Jessen M, Malm L. The importance of nasal airway resistance and nasal symptoms in the selection of patients for septoplasty. Rhinology 1984; 22:157-164.

36. Marais J, Murray JA, Marshall I, Douglas N, Martin S. Minimal cross-sectional areas, nasal peak flow and patients' satisfaction in septoplasty and inferior turbinectomy. Rhinology. 1994; 32: 145147.

37. Postema CA, Huygen PL, Lecluse RG, Wentges RT. The lateralization percentage as a measure of nasal flow asymmetry in active anterior rhinomanometry. Clin Otolaryngol Allied Sci. 1980; 5: 165-170.

\section{G. Fyrmpas}

Andrea Papandreou 96

56728 Neapoli

Thessaloniki

Greece

Tel: +30-693-243 0640

Fax: +30-231-099 4916

E-mail: drfirbas@hotmail.com 